\title{
A $d$-DIMENSIONAL EXTENSION OF A LEMMA OF HUNEKE'S AND FORMULAS FOR THE HILBERT COEFFICIENTS
}

\author{
SAM HUCKABA \\ (Communicated by Wolmer V. Vasconcelos)
}

\begin{abstract}
A $d$-dimensional version is given of a 2-dimensional result due to C. Huneke. His result produced a formula relating the length $\lambda\left(I^{n+1} / J I^{n}\right)$ to the difference $P_{I}(n+1)-H_{I}(n+1)$, where $I$ is primary for the maximal ideal of a 2-dimensional Cohen-Macaulay local ring $R, J$ is a minimal reduction of $I, H_{I}(n)=\lambda\left(R / I^{n}\right)$, and $P_{I}(n)$ is the Hilbert-Samuel polynomial of $I$. We produce a formula that is valid for arbitrary dimension, and then use it to establish some formulas for the Hilbert coefficients of $I$. We also include a characterization, in terms of the Hilbert coefficients of $I$, of the condition $\operatorname{depth}(G(I)) \geq d-1$.
\end{abstract}

\section{INTRODUCTION}

If $(R, m)$ is a $d$-dimensional (Noetherian) local ring and $I$ is an $m$-primary ideal of $R$, then let $\lambda\left(R / I^{n}\right)$ denote the length of the $R$-module $R / I^{n}$. The Hilbert-Samuel function of $I$ is the function $H_{I}(n)=\lambda\left(R / I^{n}\right)$. It follows from the theory of Hilbert functions applied to the associated graded ring $G(I)=\bigoplus_{n>0} I^{n} / I^{n+1}$ of $I$ that there is an integer-valued polynomial $P_{I}(n)$ of degree $d$ with rational coefficients such that $P_{I}(n)=H_{I}(n)$ for all $n>>0 . P_{I}(n)$ is called the Hilbert-Samuel polynomial of $I$. These are often referred to simply as the Hilbert function and Hilbert polynomial of $I$. The Hilbert polynomial of $I$ may be written as

$P_{I}(n)=e_{0}(I)\left(\begin{array}{c}n+d-1 \\ d\end{array}\right)-e_{1}(I)\left(\begin{array}{c}n+d-2 \\ d-1\end{array}\right)+\cdots+(-1)^{d-1} e_{d-1}(I) n+(-1)^{d} e_{d}(I)$

and the coefficients $e_{i}(I)$ are called the Hilbert coefficients of $I$. Of special importance is $e_{0}(I)$, which is called the multiplicity of $I$. In the geometric case and with $I=m$ it equals the multiplicity of the point corresponding to $m$ on the variety described by $R$. It has been extensively studied. The other Hilbert coefficients have not been studied in such depth, although the past several years have witnessed a handful of articles using or interpreting them (see for example [Hun], $[\mathrm{KM}],[\mathrm{K}]$, [M2], [Na], [No], [O], [S1], [S2], [V]).

Received by the editors June 8, 1994 and, in revised form, November 8, 1994.

1991 Mathematics Subject Classification. Primary 13D40, 13A30, 13 H10.

Key words and phrases. Hilbert-Samuel polynomial, depth, associated graded ring, CohenMacaulay.

The author was partially supported by the NSA (\#MDA904-92-H-3040).

(C)1996 American Mathematical Society 
In [Hun] C. Huneke gave an interesting formula involving the powers, the Hilbert function, and the Hilbert polynomial of $I$, in case $R$ is a 2-dimensional CohenMacualay local ring. Recall first that if $(R, m)$ is any $d$-dimensional local ring with $R / m$ infinite and $I$ is an $m$-primary ideal of $R$, then there exists a $d$-generated ideal $J$ of $R$ such that $J I^{n}=I^{n+1}$ for $n>0 . J$ is called a minimal reduction of $I$, and fits into a broader theory of reductions initiated in [NR]. Even if $R / m$ is not assumed to be infinite it is usually true that $I$ has a $d$-generated minimal reduction $J$, although in general such a $J$ need not exist. If $f: Z \rightarrow Z$ is a function let $\Delta$ denote the first difference function defined by $\Delta[f(n)]=f(n)-f(n-1)$, and let $\Delta^{i}$ be defined by $\Delta^{i}[f(n)]=\Delta^{i-1}[\Delta[f(n)]]$. By convention $\Delta^{0}[f(n)]=f(n)$.

Lemma 1.1 [Hun, Fundamental Lemma 2.4]. Let $(R, m)$ be a 2-dimensional Cohen-Macaulay local ring, $I$ an m-primary ideal of $R$, and $J=\left(x_{1}, x_{2}\right)$ a minimal reduction of $I$. Then

$$
\lambda\left(I^{n+1} / J I^{n}\right)-\lambda\left(\left(I^{n}: J\right) / I^{n-1}\right)=\Delta^{2}\left[P_{I}(n+1)-H_{I}(n+1)\right],
$$

for all $n \geq 1$.

Huneke used this lemma to give some formulas for the Hilbert coefficients and to characterize the condition $H_{I}(n)=P_{I}(n)$ for all $n \geq 1$ under certain restrictions on $I$. The characterization was expanded by J.D. Sally in [S1], [S2]. In this paper we prove a $d$-dimensional version of Lemma 1.1, namely that

$$
\lambda\left(I^{n+1} / J I^{n}\right)+w_{n}(J, I)=\Delta^{d}\left[P_{I}(n+1)-H_{I}(n+1)\right]
$$

for each $n \geq 0$ where $w_{n}(J, I)$ are integers and $J$ is generated by a superficial sequence (see Theorem 2.4). The reader should consult $\S 2$ for the definitions of superficial sequence and $w_{n}(J, I)$. The $w_{n}(J, I)$ are a bit complicated in the general case, but they collapse to zero in interesting special cases (see the next paragraph). Using Theorem 2.4 we are able to give formulas for the Hilbert coefficients (see Corollary 2.10).

If $M=m / I \oplus I / I^{2} \oplus I^{2} / I^{3} \oplus \ldots$ denotes the maximal homogeneous ideal of $G(I)$, then by $\operatorname{depth}(G(I))$ we mean the depth of the local ring $G(I)_{M}$. The condition $\operatorname{depth}(G(I)) \geq d-1$ on $I$ is a useful one, especially when considering questions about the behavior of the powers $I^{n}$. For example, the Hilbert coefficients have certain predictable characteristics (e.g. see [M2]). If $\operatorname{depth}(G(I)) \geq d-1$, then Corollary 2.10 may be improved (see Corollary 2.11), because $w_{n}(J, I)=0$ for all $n$ in that case. It reduces the computation of the Hilbert polynomial to a computation of the lengths of $I^{n+1} / J I^{n}$ for $n$ between 0 and the reduction number of $I$ with respect to $J$.

In $\S 3$ we show that the condition $\operatorname{depth}(G(I)) \geq d-1$ may be characterized by the behavior of $e_{1}(I)$. In particular we show in Theorem 3.1 that

$$
\sum_{n=0}^{\infty} \lambda\left(I^{n+1} / J I^{n}\right)=e_{1}(I) \text { if and only if } \operatorname{depth}(G(I)) \geq d-1 .
$$

Theorem 3.1 fits in loosely with results of A. Guerrieri [G]. She studied the quantity $\sum_{n=2}^{\infty} \lambda\left(\left(I^{n} \cap J\right) / J I^{n-1}\right)$ and related it to the condition $\operatorname{depth}(G(I)) \geq d-1$.

For $x \in R$ we let $x^{\prime}$ denote the leading form of $x$ in $G(I)$. Recall that $x \in I^{k} \backslash I^{k+1}$ is a $G(I)$-regular element if and only if $\left(I^{n+k}: x\right)=I^{n}$ for all $n \geq 0$. 


\section{MAIN RESUlT}

Let $(R, m)$ be a local ring of dimension $d>0$ with $R / m$ infinite, let $I$ be an $m$-primary ideal of $R$, and let $J=\left(x_{1}, \ldots, x_{d}\right)$ be a minimal reduction of $I$. Recall that an element $x \in I \backslash I^{2}$ is said to be superficial (of order one) for $I$ if there is a positive integer $c$ such that $\left(I^{n}: x\right) \cap I^{c}=I^{n-1}$ for all $n>c$. By the Artin-Rees lemma if $x$ is $R$-regular and superficial for $I$, then $\left(I^{n}: x\right)=I^{n-1}$ for all $n>>0$. Superficial elements for $I$ exist if $R / m$ is infinite ([N] or [ZS]). If $y_{1}, \ldots, y_{s} \in I$ such that the image of $y_{i}$ in $I /\left(y_{1}, \ldots, y_{i-1}\right)$ is superficial for $I /\left(y_{1}, \ldots, y_{i-1}\right)$ for each $i$, then $\left\{y_{1}, \ldots, y_{s}\right\}$ is called a superficial sequence for $I$. We introduce the following notation to simplify our later statements.

Definition 2.1. Let $(R, m)$ be a local ring of dimension $d>0$, let $I$ be an $m$ primary ideal of $R$, and let $J$ be a minimal reduction of $I$. Assume $J=\left(x_{1}, \ldots, x_{d}\right)$ where $\left\{x_{1}, \ldots, x_{d}\right\}$ is a superficial sequence for $I$, and let $J_{i}=\left(x_{1}, \ldots, x_{i}\right)$ for $0 \leq i \leq d$ where $J_{0}=(0)$. For $n$ a non-negative integer, define $w_{n}(J, I)$ by $w_{n}(J, I)=0$ if $d=1$, and for $d>1$ by

$$
\begin{aligned}
w_{n}(J, I) & =\Delta^{d-1}\left[\lambda\left(\left(I^{n+1}: x_{1}\right) / I^{n}\right)\right]+\Delta^{d-2}\left[\lambda\left(\left(\left(I^{n+1}, J_{1}\right): x_{2}\right) /\left(I^{n}, J_{1}\right)\right)\right]+\ldots \\
& +\Delta\left[\lambda\left(\left(\left(I^{n+1}, J_{d-2}\right): x_{d-1}\right) /\left(I^{n}, J_{d-2}\right)\right)\right] \\
& -\lambda\left(\left(I^{n+1}: x_{1}\right) /\left(J I^{n}: x_{1}\right)\right)-\lambda\left(\left(\left(I^{n+1}, J_{1}\right): x_{2}\right) /\left(\left(J I^{n}, J_{1}\right): x_{2}\right)\right)-\ldots \\
& -\lambda\left(\left(\left(I^{n+1}, J_{d-2}\right): x_{d-1}\right) /\left(\left(J I^{n}, J_{d-2}\right): x_{d-1}\right)\right) .
\end{aligned}
$$

Remark 2.2. In Definition 2.1 it holds, by using that $\left\{x_{1}, \ldots, x_{d}\right\}$ is a superficial sequence for $I$, that $w_{n}(J, I)=0$ for $n>>$. If $R / m$ is infinite, then we may assume that $J=\left(x_{1}, \ldots, x_{d}\right)$ is generated by a superficial sequence for $I$ (see [Wu, Remark 2.6]). Conversely, any $d$-generated superficial sequence for $I$ will generate a minimal reduction of $I$ (see [Sw, Lemma 1.1]).

Lemma 2.3. If $R, I$, and $J=\left(x_{1}, \ldots, x_{d}\right)$ are as in Definition 2.1 and if $d>1$, then

$$
w_{n}(J, I)=w_{n}(\bar{J}, \bar{I})+\Delta^{d-1}\left[\lambda\left(\left(I^{n+1}: x_{1}\right) / I^{n}\right)\right]-\lambda\left(\left(I^{n+1}: x_{1}\right) /\left(J I^{n}: x_{1}\right)\right)
$$

for all $n \geq 1$, where " - " denotes image modulo $\left(x_{1}\right)$.

Proof. This is clear because $x_{1} \in\left(I^{n}, J_{i}\right)$ and $x_{1} \in\left(\left(J I^{n}, J_{i}: x_{i+1}\right)\right.$ for all $i \geq 1$.

The following is our main result.

Theorem 2.4. Let $(R, m)$ be a Cohen-Macaulay local ring of dimension $d>0$, let $I$ be an m-primary ideal of $R$, let $J=\left(x_{1}, \ldots, x_{d}\right)$ be a minimal reduction of $I$, and assume $\left\{x_{1}, \ldots, x_{d}\right\}$ is a superficial sequence for $I$. Then for all $n \geq 0$,

$$
\lambda\left(I^{n+1} / J I^{n}\right)+w_{n}(J, I)=\Delta^{d}\left[P_{I}(n+1)-H_{I}(n+1)\right] .
$$

Proof. Note that $\left\{x_{1}, \ldots, x_{d}\right\}$ is an $R$-regular sequence because $R$ is CohenMacaulay. The proof is by induction on $d$. If $d=1$, then we have $w_{n}(J, I)=0$ and $\lambda\left(I^{n+1} / x_{1} I^{n}\right)=\lambda\left(R /\left(x_{1}\right)\right)+\lambda\left(\left(x_{1}\right) / x_{1} I^{n}\right)-\lambda\left(R / I^{n+1}\right)$. Since $\left(x_{1}\right)$ is a minimal reduction of $I$ and $R$ is Cohen-Macaulay, $e_{0}(I)=\lambda\left(R /\left(x_{1}\right)\right)$. Using that $x_{1}$ is $R$ - 
regular we see that $\left(x_{1}\right) / x_{1} I^{n} \cong R / I^{n}$. Therefore $\lambda\left(I^{n+1} / x_{1} I^{n}\right)=e_{0}(I)+H_{I}(n)-$ $H_{I}(n+1)$, which is easily seen to equal $\Delta\left[P_{I}(n+1)-H_{I}(n+1)\right]$.

Let $d>1$ and let "- " denote image modulo $\left(x_{1}\right)$. We have

$$
\begin{aligned}
\lambda\left(I^{n+1} / J I^{n}\right)= & \lambda\left(R /\left(J I^{n}, x_{1}\right)\right)+\lambda\left(\left(J I^{n}, x_{1}\right) / J I^{n}\right)-\lambda\left(R /\left(I^{n+1}, x_{1}\right)\right) \\
& -\lambda\left(\left(I^{n+1}, x_{1}\right) / I^{n+1}\right) .
\end{aligned}
$$

Using that $\left(J I^{n}, x_{1}\right) / J I^{n} \cong R /\left(J I^{n}: x_{1}\right)$ and $\left(I^{n+1}, x_{1}\right) / I^{n+1} \cong R /\left(I^{n+1}: x_{1}\right)$ we obtain

$$
\begin{aligned}
\lambda\left(I^{n+1} / J I^{n}\right) & =\lambda\left(\overline{I^{n+1}} / \overline{J I^{n}}\right)+\lambda\left(R /\left(J I^{n}: x_{1}\right)\right)-\lambda\left(R /\left(I^{n+1}: x_{1}\right)\right) \\
& =\lambda\left(\overline{I^{n+1}} / \overline{J I^{n}}\right)+\lambda\left(\left(I^{n+1}: x_{1}\right) /\left(J I^{n}: x_{1}\right)\right) .
\end{aligned}
$$

Combining this with the induction hypothesis and Lemma 2.3 we obtain

$\lambda\left(I^{n+1} / J I^{n}\right)+w_{n}(J, I)=\Delta^{d-1}\left[P_{\bar{I}}(n+1)-H_{\bar{I}}(n+1)\right]+\Delta^{d-1}\left[\lambda\left(\left(I^{n+1}: x_{1}\right) / I^{n}\right)\right]$.

Note that $P_{\bar{I}}(n+1)=P_{I}(n+1)-P_{I}(n)$ because $x_{1}$ is superficial for $I$, and that $H_{\bar{I}}(n+1)=H_{I}(n+1)-H_{I}(n)+\lambda\left(\left(I^{n+1}: x_{1}\right) / I^{n}\right)$ by $([\mathrm{N},(22.6)])$. Therefore

$$
\begin{aligned}
\lambda\left(I^{n+1}\right. & \left./ J I^{n}\right)+w_{n}(J, I) \\
= & \Delta^{d-1}\left[\Delta\left[P_{I}(n+1)-H_{I}(n+1)\right]-\lambda\left(\left(I^{n+1}: x_{1}\right) / I^{n}\right)\right] \\
& +\Delta^{d-1}\left[\lambda\left(\left(I^{n+1}: x_{1}\right) / I^{n}\right)\right] \\
= & \Delta^{d}\left[P_{I}(n+1)-H_{I}(n+1)\right],
\end{aligned}
$$

completing the proof of Theorem 2.4.

Remark 2.5. The case $d=2$ of Theorem 2.4 is Huneke's lemma as follows: First note that

$$
\begin{aligned}
w_{n}(J, I) & =\Delta\left[\lambda\left(\left(I^{n+1}: x_{1}\right) / I^{n}\right)\right]-\lambda\left(\left(I^{n+1}: x_{1}\right) /\left(J I^{n}: x_{1}\right)\right) \\
& =\lambda\left(\left(J I^{n}: x_{1}\right) / I^{n}\right)-\lambda\left(\left(I^{n}: x_{1}\right) / I^{n-1}\right) .
\end{aligned}
$$

Next, observe that

$$
\left(J I^{n}: x_{1}\right) / I^{n} \cong\left(I^{n}: x_{1}\right) /\left(I^{n}: J\right) .
$$

This isomorphism follows by standard isomorphism theorems, using that $\left\{x_{1}, x_{2}\right\}$ is an $R$-sequence, along with the equality $x_{1} I^{n} \cap x_{2} I^{n}=x_{1} x_{2}\left(I^{n}: J\right)$ (which is valid because $\left\{x_{1}, x_{2}\right\}$ is an $R$-sequence). By taking lengths it follows that $w_{n}(J, I)=$ $-\lambda\left(\left(I^{n}: J\right) / I^{n-1}\right)$ which yields Huneke's lemma. Observe that Theorem 2.4 implies the independence of $w_{n}(J, I)$ from the choice of superficial sequence generating $J$. Note also that $w_{n}(J, I) \leq 0$ in the 2-dimensional case. If $d>2$, and one is willing to accept calculations using Macaulay, then evidence is strong that it is possible to have $w_{n}(J, I)>0$. As an example we offer the following taken from A. Guerrieri's thesis [G]: Let $T=k[x, y, z], N=(x, y, z)$, and $R=T_{N}$, where $k=Z /(31,991) Z$, and let $I=\left(x^{3}, y^{3}, x^{2} y+z^{3}, x z^{2}, y^{2} z+x^{2} z\right) R$. By using Macaulay to generate a random minimal reduction $J=\left(x_{1}, x_{2}, x_{3}\right)$ one finds that $w_{3}(J, I)=1$. This is not a genuine example of course, even modulo Macaulay, unless one can verify 
that $\left\{x_{1}, x_{2}, x_{3}\right\}$ is a superficial sequence for $I$. From the theory of reductions and superficial elements however, any sufficiently general sequence of three elements (obtained as linear combinations of the 5 generators of $I$ ) will form a superficial sequence for $I$ and a minimal reduction of $I$. Specifically, if $x_{1}$ is chosen so that $x_{1}^{\prime} \notin \cup\left\{P \in \operatorname{Ass}(G(I)) \mid G(I)^{+} \nsubseteq P\right\}$, then $x_{1}$ is superficial for $I$ (see [N, $\left.\S 22\right]$ ). If the residue field is 'large enough' in relation to the cardinality of $\operatorname{Ass}(G(I))$, then the set of linear combinations will contain a superficial element. It follows from [Sw, Lemma 1] that a maximal superficial sequence for $I$ generates a minimal reduction of $I$. Therefore the probability is quite high that a randomly chosen sequence $\left\{x_{1}, x_{2}, x_{3}\right\}$ will satisfy the condition.

In the event that $\operatorname{depth}(G(I) \geq d-1$ we obtain the best possible situation, namely that $w_{n}(J, I)=0$ for all $n \geq 0$ (and, if $R / m$ is infinite, for any choice of $J)$. The next corollary spells out a consequence of this. It was initially discovered by T. Marley using different techniques.

Corollary 2.6 ([M2, Corollary 3.9]). If $R$ and I are as in Theorem 2.4, depth $(G(I))$ $\geq d-1$, and $J$ is a minimal reduction of $I$, then

$$
\lambda\left(I^{n+1} / J I^{n}\right)=\Delta^{d}\left[P_{I}(n+1)-H_{I}(n+1)\right] .
$$

In particular, $\lambda\left(I^{n+1} / J I^{n}\right)$ is independent of $J$.

Proof. We may assume that $J=\left(x_{1}, \ldots, x_{d}\right)$ where $\left\{x_{1}^{\prime}, \ldots, x_{d-1}^{\prime}\right\}$ is a $G(I)$-regular sequence, thus $w_{n}(J, I)=0$ for all $n \geq 0$.

Next we establish formulas for the Hilbert coefficients of an $m$-primary ideal $I$ of a Cohen-Macaulay local ring $(R, m)$. These formulas are extensions of those given in [Hun] for dimension 2. The following lemma provides a useful identity. For convenience we include the proof, which amounts to a routine summation by parts.

Lemma 2.7. Let $f: Z \rightarrow Z$ be a function such that $f(n)=0$ for $n>>0$. Then the following two statements are true.

(1) For all $k, j, 0<k \leq d, 0<j \leq d$,

$$
\sum_{n=k}^{\infty}\left(\begin{array}{l}
n \\
k
\end{array}\right) \Delta^{j}[f(n+1)]=-\sum_{n=k-1}^{\infty}\left(\begin{array}{c}
n \\
k-1
\end{array}\right) \Delta^{j-1}[f(n+1)] .
$$

(2) If $f(n)=0$ for all $n \leq 0$, then $\sum_{n=0}^{\infty} \Delta^{j}[f(n+1)]=0$ for $0<j \leq d$.

Proof. For all $k \geq 1$ we have

$$
\begin{aligned}
\sum_{n=k}^{\infty}\left(\begin{array}{l}
n \\
k
\end{array}\right) \Delta^{j}[f(n+1)] & =\sum_{n=k}^{\infty}\left(\begin{array}{l}
n \\
k
\end{array}\right) \Delta^{j-1}[f(n+1)-f(n)] \\
& =\sum_{n=k}^{\infty}\left(\begin{array}{l}
n \\
k
\end{array}\right) \Delta^{j-1}[f(n+1)]-\sum_{n=k-1}^{\infty}\left(\begin{array}{c}
n+1 \\
k
\end{array}\right) \Delta^{j-1}[f(n+1)] \\
& =-\Delta^{j-1}[f(k)]-\sum_{n=k}^{\infty}\left(\begin{array}{c}
n \\
k-1
\end{array}\right) \Delta^{j-1}[f(n+1)] \\
& =-\sum_{n=k-1}^{\infty}\left(\begin{array}{c}
n \\
k-1
\end{array}\right) \Delta^{j-1}[f(n+1)] .
\end{aligned}
$$


To prove the second statement note that $\sum_{n=0}^{\infty} \Delta[f(n+1)]=-f(0)$, hence

$$
\sum_{n=0}^{\infty} \Delta^{j}[f(n+1)]=-\Delta^{j-1}[f(0)]
$$

The condition $f(n)=0$ for all $n \leq 0$ finishes the proof.

The next lemma is essentially well known (see [BH, Section 4.1]). For convenience we include a proof.

Lemma 2.8. Let $(R, m)$ be a local ring of dimension $d>0$ and let $I$ be an $m$ primary ideal of $R$. Assume that $R$ contains a superficial sequence of length $d$ for $I$. If $P_{I}(n)$ is the Hilbert polynomial of $I$, then $\Delta^{d-i}\left[P_{I}(0)\right]=(-1)^{i} e_{i}(I)$ for $1 \leq i \leq d$.

Proof. The proof is by induction on $d$. Let $i$ be an integer such that $1 \leq i \leq d$. If $d=i$, then the result is clear. Suppose $i<d$ and let $x$ be a superficial element for I. Then $\Delta^{d-i}\left[P_{I}(0)\right]=\Delta^{(d-1)-i}\left[P_{\bar{I}}(0)\right]=(-1)^{i} e_{i}(\bar{I})=(-1)^{i} e_{i}(I)$, the first and last equalities because $x$ is superficial for $I$.

Proposition 2.9. Let $(R, m)$ be a local ring of dimension $d>0$ and let $I$ be an $m$-primary ideal of $R$. Assume that $R$ contains a superficial sequence of length $d$ for I. Then $\sum_{n=i-1}^{\infty}\left(\begin{array}{c}n \\ i-1\end{array}\right) \Delta^{d}\left[P_{I}(n+1)-H_{I}(n+1)\right]=e_{i}(I)$ for $1 \leq i \leq d$.

Proof. Set $f(n)=P_{I}(n)-H_{I}(n)$. Let $i$ be an integer such that $1 \leq i \leq d$. By repeated use of Lemma 2.7(1) we obtain

$$
\sum_{n=i-1}^{\infty}\left(\begin{array}{c}
n \\
i-1
\end{array}\right) \Delta^{d}[f(n+1)]=(-1)^{i-1} \sum_{n=0}^{\infty} \Delta^{d-i+1}[f(n+1)],
$$

and

$$
\begin{aligned}
(-1)^{i-1} \sum_{n=0}^{\infty} \Delta^{d-i+1}[f(n+1)] & =(-1)^{i-1} \sum_{n=0}^{\infty} \Delta^{d-i+1}\left[P_{I}(n+1)\right] \\
& =(-1)^{i} \Delta^{d-i}\left[P_{I}(0)\right]=e_{i}(I),
\end{aligned}
$$

using Lemma 2.7(2) for the first equality and Lemma 2.8 for the last equality.

We can use Proposition 2.9 to extend the formulas [Hun, Remarks 2.7,2.8] to arbitrary dimension. Notice that the choice of a superficial sequence below insures that the sums are actually finite.

Corollary 2.10. Let $(R, m)$ be a Cohen-Macaulay local ring of dimension $d>0$ and let $I$ be an $m$-primary ideal of $R$. Let $J$ be a minimal reduction of $I$ and assume that $J$ is generated by a superficial sequence of length $d$ for $I$. Let $v_{n}(J, I)=$ $\lambda\left(I^{n+1} / J I^{n}\right)+w_{n}(J, I)$. Then the Hilbert coefficients of I satisfy the formulas $\sum_{n=i-1}^{\infty}\left(\begin{array}{c}n \\ i-1\end{array}\right) v_{n}(J, I)=e_{i}(I)$ for $1 \leq i \leq d$.

Proof. Immediate from Theorem 2.4 and Proposition 2.9. 
Corollary 2.11. Let $R, I$, and $J$ be as in Corollary 2.10, and assume that $\operatorname{depth}(G(I)) \geq d-1$. Then $\sum_{n=i-1}^{\infty}\left(\begin{array}{c}n \\ i-1\end{array}\right) \lambda\left(I^{n+1} / J I^{n}\right)=e_{i}(I)$ for $1 \leq i \leq d$.

Proof. Use Corollary 2.6 and Proposition 2.9.

In the next section we will discuss a converse to Corollary 2.11. Note that Corollary 2.11 may be used in conjunction with Macaulay to find $P_{I}(n)$ for appropriate ideals $I$.

Example 2.12. Let $T=k[x, y, z]$ (where $k=Z /(31,991) Z), N=(x, y, z) T$, $R=T_{N}$, and $I=\left(x^{2}, y^{3}, z^{3}, x^{2} z+y z^{2}\right)$. Then $\operatorname{depth}(G(I))=2$ and, by computing lengths and using Corollary 2.11, $P_{I}(n)=27\left(\begin{array}{c}n+2 \\ 3\end{array}\right)-18\left(\begin{array}{c}n+1 \\ 2\end{array}\right)+31 n-56$.

\section{An Application to $\operatorname{depth}(G(I)) \geq d-1$}

We present here a converse to Corollary 2.11. This serves as a characterization of the condition $\operatorname{depth}(G(I)) \geq d-1$ in case $(R, m)$ is Cohen-Macaulay and $I$ is $m$-primary. It was originally discovered only for small $d$. The author thanks Irena Swanson for providing the proof for arbitrary $d$, and the referee for pointing out a further simplification in the proof.

Theorem 3.1. Let $(R, m)$ be a Cohen-Macaulay local ring of dimension d, let I be an m-primary ideal of $R$, let $J$ be a minimal reduction of $I$, and assume that $J$ is generated by a superficial sequence of length $d$ for $I$. Then the following two statements are equivalent.

(1) $\sum_{n=0}^{\infty} \lambda\left(I^{n+1} / J I^{n}\right)=e_{1}(I)$.

(2) $\operatorname{depth}(G(I)) \geq d-1$.

Proof. Corollary 2.11 yields that (2) implies (1). Assume (1) holds and let $J=$ $\left(x_{1}, \ldots, x_{d}\right)$ be a minimal reduction of $I$ where $\left\{x_{1}, \ldots, x_{d}\right\}$ is a superficial sequence for $I$. We will show that $\left\{x_{1}^{\prime}, \ldots, x_{d-1}^{\prime}\right\}$ is a $G(I)$-regular sequence. By Corollary 2.10 we have $\sum_{n=0}^{\infty} w_{n}(J, I)=0$. Note that by Lemma $2.7(2)$

$$
\begin{aligned}
\sum_{n=0}^{\infty} w_{n}(J, I) & =-\sum_{n=0}^{\infty} \lambda\left(\left(I^{n+1}: x_{1}\right) /\left(J I^{n}: x_{1}\right)\right) \\
& -\ldots-\sum_{n=0}^{\infty} \lambda\left(\left(\left(I^{n+1}, J_{d-2}\right): x_{d-1}\right) /\left(\left(J I^{n}, J_{d-2}\right): x_{d-1}\right)\right)
\end{aligned}
$$

therefore

$$
\left(\left(I^{n+1}, J_{i-1}\right): x_{i}\right)=\left(\left(J I^{n}, J_{i-1}\right): x_{i}\right)
$$

for all $n \geq 0$ and $1 \leq i \leq d-1$. We now prove by induction on $n$ that $J_{d-1} \cap I^{n+1}=$ $J_{d-1} I^{n}$ for all $n \geq 0$. The case $n=0$ is clear. Let $n>0$ and let $r \in J_{d-1} \cap I^{n+1}$. Write $r=\sum_{i=1}^{d-1} r_{i} x_{i}$ with $r_{i} \in R$. Then $r_{d-1} \in\left(\left(I^{n+1}, J_{d-2}\right): x_{d-1}\right)=\left(\left(J I^{n}, J_{d-2}\right)\right.$ : $\left.x_{d-1}\right)$ by (3.2), hence $r_{d-1} x_{d-1}=\sum_{i=1}^{d} a_{i} x_{i}+\sum_{i=1}^{d-2} b_{i} x_{i}$ for some $a_{i} \in I^{n}, b_{i} \in R$. 
Because $\left\{x_{1}, \ldots, x_{d}\right\}$ is a regular sequence, we have $a_{d} \in J_{d-1}$. Thus $a_{d} \in J_{d-1} \cap I^{n}=$ $J_{d-1} I^{n-1}$ by induction, hence we may write $a_{d}=\sum_{i=1}^{d-1} c_{i} x_{i}$ for some $c_{i} \in I^{n-1}$. Substituting into the equation for $r$ we obtain $r=\sum_{i=1}^{d-2}\left(r_{i}+b_{i}\right) x_{i}+\sum_{i=1}^{d-1}\left(a_{i}+c_{i} x_{d}\right) x_{i}$ where $r_{i}+b_{i} \in R$ and $a_{i}+c_{i} x_{d} \in I^{n}$. If $s=\sum_{i=1}^{d-1}\left(a_{i}+c_{i} x_{d}\right) x_{i}$, then $r-s=$ $\sum_{i=1}^{d-2}\left(r_{i}+b_{i}\right) x_{i}$ and $s \in J_{d-1} I^{n}$. Therefore $r-s \in J_{d-2} \cap I^{n+1}$. We may now repeat the argument for $r-s$ and find $s^{\prime} \in J_{d-1} I^{n}$ such that $r-s^{\prime} \in J_{d-3} \cap I^{n+1}$. Continuing we eventually obtain that $r \in J_{d-1} I^{n}$. By using [VV, Corollary 2.7] we conclude that $\left\{x_{1}^{\prime}, \ldots, x_{d-1}^{\prime}\right\}$ is a $G(I)$-regular sequence, completing the proof.

\section{ACKNOWLEDGMENT}

I wish to thank Irena Swanson for pointing out the correct induction argument for the proof of Theorem 3.1. Her suggestion extended the original form, which was for $d \leq 3$, to arbitrary dimension $d$.

\section{REFERENCES}

[BH] W. Bruns and J. Herzog, Cohen-Macaulay Rings, Cambridge Studies in Advanced Mathematics, vol. 39, Cambridge University Press, Cambridge, 1993. CMP 94:05

[G] A. Guerrieri, On the depth of certain graded rings associated to an ideal, Ph.D. Dissertation, Purdue University (1993).

[Hun] C. Huneke, Hilbert functions and symbolic powers, Michigan Math. J. 34 (1987), 293-318. MR 89b: 13037

[KM] D. Kirby and H. A. Mehran, A note on the coefficients of the Hilbert-Samuel polynomial for a Cohen-Macaulay module, J. London Math. Soc. (2) 25 (1982), 449-457. MR 84a:13022

[K] K. Kubota, On the Hilbert-Samuel function, Tokyo J. Math. 8 (1985), 439-448. MR 87f:13023a

[M1] T. Marley, Hilbert functions of ideals in Cohen-Macaulay local rings, Ph.D. Dissertation, Purdue University (1989).

[M2] T. Marley, The coefficients of the Hilbert polynomial and the reduction number of an ideal, J. London Math. Soc. (2) 40 (1989), 1-8. MR 90m:13026

[N] M. Nagata, Local Rings, Kreiger, Huntington and New York, 1975. MR 57:301

[Na] M. Narita, A note on the coefficients of Hilbert characteristic functions in semi-regular local rings, Proc. Cambridge Philos. Soc. 59 (1963), 269-275. MR 26:3734

[No] D. G. Northcott, A note on the coefficients of the abstract Hilbert function, J. London Math. Soc. 35 (1960), 209-214. MR 22:1599

[NR] D. G. Northcott and D. Rees, Reductions of ideals in local rings, Proc. Cambridge Philos. Soc. 50 (1954), 145-158. MR 15:596a

[O] A. Ooishi, $\Delta$-genera and sectional genera of commutative rings, Hiroshima Math. J. 17 (1987), 361-372. MR 89f: 13033

[S1] J. D. Sally, Hilbert coefficients and reduction number 2, J. Algebraic Geom. 1 (1992), 325-333. MR 93b:13026

[S2] J. D. Sally, Ideals whose Hilbert function and Hilbert polynomial agree at $n=1$, J. Algebra 157 (1993), 534-547. MR 94d:13016

[Sw] I. Swanson, A note on analytic spread, Comm. Algebra 22 (1994), 407-411. MR 95b:13007

[VV] P. Valabrega and G. Valla, Form rings and regular sequences, Nagoya Math. J. 72 (1978), 93-101. MR 80d:14010

[V] W. V. Vasconcelos, Hilbert functions, analytic spread, and Koszul homology, Contemporary Math. 159 (1994), 401-422. MR 95a:13006 
[Wu] Y. Wu, Reduction numbers and Hilbert polynomials of ideals in higher dimensional Cohen-Macaulay local rings, Math. Proc. Cambridge Philos. Soc. 111 (1992), 47-56. MR 92g: 13020

[ZS] O. Zariski and P. Samuel, Commutative Algebra, vol. 2, Springer-Verlag, New York, 1960. MR 52:10706

Department of Mathematics, Florida State University, Tallahassee, Florida 32306 3027

E-mail address: huckaba@math.fsu.edu 